\title{
ARTIST: A computer system for creating and running psychology experiments
}

\author{
DIANA EUGENIE KORNBROT \\ The Hatfield Polytechnic, Hatfield, AL109AB, England
}

\begin{abstract}
A flexible computer system for research and teaching experiments in psychology is described. Special features of the system include (1) separate phases for the generation and running of experiments, (2) facilities to control presentation of stimuli according to any factorial design, and (3) different modes of operation depending on the prior knowledge of the users. The system is evaluated both in terms of the range of experiments that can be performed and in terms of the time and effort required to create new experiments or modify old ones. General principles for designing interactive systems are discussed in the light of experiences with this particular system.
\end{abstract}

ARTIST is a laboratory computer system for creating and running psychological experiments in cognition and perception. The system is command based. The user communicates with the system by typing a four-letter command followed by parameters. ARTIST is designed to be used by people with very different levels of both computer and psychology knowledge.

The major concern of this paper is to describe how ARTIST actually works as a laboratory tool in a typical British psychology department. The first section sets out the goals that ARTIST was designed to achieve. The following sections describe the workings of the system, details of the implementation, and evaluation of the actual use of the system by groups of different degrees of sophistication. The final section assesses that part of the work on ARTIST that can be used outside our own department and discusses the general lessons to be learned about designing systems for "specialist" users. (The term "specialist" describes computer users who have considerable expertise in some domain-here, psychological experiments-but little knowledge or interest in the details of computer systems.) It is hoped that the discussion of ARTIST may be useful to computer scientists, as well as to psychologists, in that it will give a feel for the problem as seen by actual users.

\section{DESIGN GOALS FOR ARTIST}

The main goal is that the system should provide a powerful and usable tool for a very wide range of experiments in perception and cognition. This goal may be loosely divided into experimental subgoals concerned

We thank Noel Lawler, chief psychology technician, for creating interfaces and maintaining the computer laboratory, Dave Miller of the Computer Centre for writing the peripheral control sof tware, and Richard Davis for evaluation of the documentation. Requests for reprints should be addressed to D. E. Kornbrot, Psychology Group, The Hatfield Polytechnic, P.O. Box 109, Hatfield, Hertfordshire, U.K. with power and range and user subgoals concerned with simplicity of operation.

\section{Experimental Goals}

The system should be able to generate as well as control any experiment consisting of a series of trial events in which each trial consists of stimulus presentation, response(s), and outcome or feedback. The stimulus display may be simple, or composite in time or space, and all three factors may be contingent on the results of previous trials. The stimulus and outcome must be selectable from a set of possible events according to an experimental design specified by the investigator.

In the generation phase, the experimenter should be able to create different displays until the final picture is satisfactory. Furthermore, the experimenter should be able to vary the timing of chosen displays and the responses that are available until the best parameters for a particular experiment are found.

In the run phase, a preprepared experiment should be both simple and quick to set up. In practice there are three stages: First, the preprepared data files are read into the computer. Second, information identifying the experiment, condition, subject, and so on, is typed in. Finally, a command is issued to "run" the experiment, that is, to present the stimuli, record the data, and summarize the results.

\section{Goals for Simplicity of Operation by Psychology Specialists}

The first goal is that a totally naive user should be able to use the system by following a few lines of instruction. At this level, subjects can be their own experimenter, or nonpsychology students (e.g., linguists) can, for example, play with "drawing" pictures or timing themselves on an arpeggio in which they successively hit up to 10 keys.

At the next level of sophistication, the user will have had some experience of using packages on a timesharing 
system (for example, statistical packages). At this level, the user knows how to create data files using an editor and how to copy and combine these files. However, such users typically have written, at most, a 20- to 50-line program in FORTRAN, and many of them will not know any programming language at all. The majority of our users fall into this category, and they should be able to go further than the naive user by modifying existing data files or creating new ones using an editor.

The most sophisticated users are themselves able to program in FORTRAN. For them, the goal is to ensure that they only have to write programs for the part of their experiment that really is unique to their application.

A psychologist well acquainted with the system should be able to set up an experiment in one or two half-day sessions, either by creating data files or by writing relatively few lines of FORTRAN.

\section{THE ARTIST SYSTEM AND ITS USERS}

\section{The Physical System}

The computer is a Digital Equipment Corporation (DEC) PDP-11/03C laboratory computer with 8-in. floppy disks for storage of data and programs. The system is controlled via a fast visual display unit ( 4,800 baud), and in addition, there is a line printer to provide a permanent record of results. The current implementation uses a Tektronix 611 oscilloscope to display tèxt and line drawings as stimuli. The 611 can display either in refresh mode with a dot writing time of 3 microsec and a consequent flicker problem for more than 12 characters or 100 lines, or in storage mode, in which the only method of clearing the screen is a 250$\mathrm{msec}$ flash. Onset and offset of the displays, response times, and interresponse times are all measured to within $1 \mathrm{msec}$.

The minimum time gap between successive displays is also $1 \mathrm{msec}$. A 16-bit parallel interface is used for responses. The physical response manipulandum has 10 keys that sit comfortably under the fingers of the two hands and an additional two slots into which any switch closure device may be plugged (e.g., voiceoperated relay, foot pedal, specially sensitive pairs of keys for forefingers, etc.). The computer may also be linked via a 1,200-baud serial link to the Polytechnic's main timesharing computer, a DEC system 1091, which supports all major statistical packages (SPSS, BMD, etc.).

\section{Users}

The users are predominantly members of the Hatfield Polytechnic Psychology Group, consisting of students enrolled in the BSc (Honours) psychology degree (roughly equivalent to American honors majors), postgraduate students reading for $\mathrm{MPhil} / \mathrm{PhD}$, and members of staff. All undergraduates attend a compulsory 14 -week course on computing in their 1st year, in which they are taught FORTRAN and how to use packages and systems facilities on the main timesharing system. They are introduced to ARTIST as a demonstration at this stage. At least one of the compulsory 2nd-year laboratory exercises is performed on the ARTIST computer system. Some of the 30 or so final-(3rd-)year students use the system for their independent research projects. The BSc students fall predominantly into the second level of sophistication described above. Postgraduates and staff vary far more widely in their expertise, but anyone who is interested can reach the second level of sophistication quickly by attending short courses or learning in apprenticeship mode from colleagues.

\section{Framework for Describing Experiments}

The psychologist, as specialist, thinks of an experiment in terms of stimuli, responses, outcomes, and experimental design, whereas the computer programmer thinks in terms of displaying a specified picture for a specified time with specified response keys active. The command structure of the system has been designed so that psychologists give all the necessary specifications to the system, but, as far as possible, in their own terms. Thus, to set up an experiment the user has to give the following information to ARTIST: (1) a list of all the text stimuli in groups of up to 12 characters, given via the command LIST (characters may be upper- or lowercase letters, numerals, and a few special symbols);(2) a list of the names of all the picture stimuli (line drawings) via the command PILI (picture list); and (3) lists of all other parameters via command TRAN (transformation), including (a) all time settings, which may be either fixed display periods or intervals or random times varying between two given limits, (b) all key settings used in the experiment, (c) all picture transformations used in the experiment (i.e., size, location, and reflection axis), and (d) a specification of the experimental design in terms of how to randomly choose indexes that specify which cell of the design to use on any particular trial. For example, in a 3 by 4 design in which the first factor is picture identity and the second factor is picture size, it may be required that in a block of 15 trials each picture appear five times and that within those five presentations Sizes 1, 2, and 3 appear once but Size 4 appear twice. All the design information needed is specified in a constraint matrix, $\mathrm{N}_{\mathrm{JI}}$, via TRAN.

The subroutine SELECT is used to choose indexes according to constraints in $\mathrm{N}_{\mathrm{JI}}$ without replacement. $\mathrm{N}_{\mathrm{J} 1}$ is the number of levels of Factor $\mathrm{J}, \mathrm{N}_{\mathrm{J} 2}$ is the cycle length in which Factor $J$ is randomized, and $\mathrm{N}_{\mathrm{J}(\mathrm{I}+2)}$ is the number of times the Ith level of Factor $\mathrm{J}$ is to be chosen within a cycle. Thus, for example, the matrix

$$
\begin{array}{llllll}
3, & 15, & 5, & 5, & 5 & \\
4, & 15, & 3, & 3, & 3, & 6
\end{array}
$$

can be used to choose an integer between 1 and 3 to specify picture identity (Factor 1 ) and a number 
between 1 and 4 to specify picture size (Factor 2). The above matrix gives an unnested design, as picture identity and picture size are chosen independently. The matrix

$\begin{array}{rrrrrr}3, & 15, & 5, & 5, & 5 & \\ 4, & 5, & 1, & 1, & 1, & 2 \\ 4, & 5, & 1, & 1, & 1, & 2 \\ 4, & 5, & 1, & 1, & 1, & 2\end{array}$

can be used for a nested design with the first row used to choose a picture identity number, $k$, and the $k+1$ th row used to choose size, thus ensuring a separate tally of size presentations for each of the three pictures. In all the above examples, the randomization takes place within a cycle of length 15 . Obviously, many designs are possible with such a system.

The graphics routines use a picture store and display buffer for the command STIM. This buffer may be

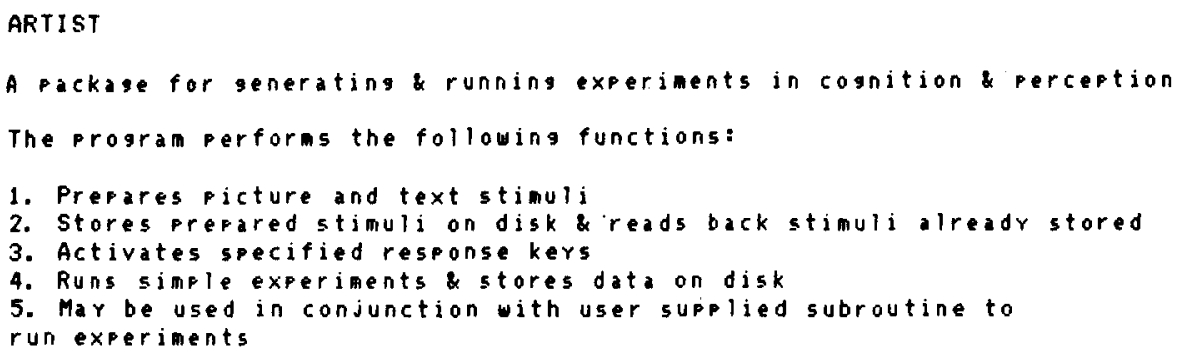

B. STIMULLIS GENEFATION

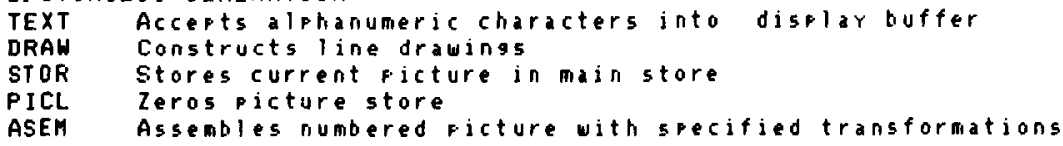

E. INTERACTION WITH IIISK

SAVE Saves specified picture on disk

REAII Feads back sfecified picture from disk

Figure 1. Introduction to ARTIST system and functional list of commands. Material that appears on a single index card is delimited by horizontal lines. 
changed using TEXT to enter characters or DRAW to create a new picture. It may also be modified by the command ASEM, which brings a numbered picture from the picture store to the buffer with specified transformations as to size, location, and reflection axis, either adding to or replacing the existing display. Thus a composite display of picture segments and/or text may be created. Mixtures of character sizes are also possible. The pictures may be transferred between the computer store and disk using READ and SAVE.

The commands TRAN, PILI, and LIST will all take input either directly from the terminal or from a named file on disk. The files that serve as input to these three commands are called specification files (they have names ending in EXP); they may be easily created and modified using the powerful system editor.

The flexibility of the ARTIST system is achieved by having a constant component common to all users and a short-variable component in a subroutine CHOICE that is specific to a particular paradigm. The paradigm implemented in the training and demonstration version is called AML (for Mueller-Lyer, one of the experiments) and consists of a stimulus presentation (drawing or text), followed by subject response, followed by feedback as to the stimulus presented. A very large range of different experiments may be implemented using this paradigm by simply changing the specification files.

\section{Using the System}

Instructions for setting up the computer and operating the ARTIST system, including three simple demonstration experiments, are available on a $5 \times 8$ in. card index system. The users may obtain copies of the information by printing out a file, ARTIST.DOC. This paper documentation consists of (1) general introduction, including a full list of commands arranged according to their functions (two index cards, as shown in Figure 1), (2) the function of each command and the information (parameters) the user must give for that command, with a separate flagged index card for each command (examples of cards for commands DRAW, STOR, SAVE are shown in Figure 2), (3) instructions for running the demonstration experiment, including one short card for starting the system and one card for each demonstration (the general start card and the card for the Mueller-Lyer illusion demonstration are shown in Figure 3), (4) a list of subroutines and their functions, as in Table 1, and (5) commented program listings.

A naive or first-time user might come on the system and draw a picture of, say, a horizontal line below a vertical line and save the picture on disk for future use. The dialogue is shown in Figure 4, with comments as to the results of each action. Such users might also run themselves on one of the demonstration experiments.

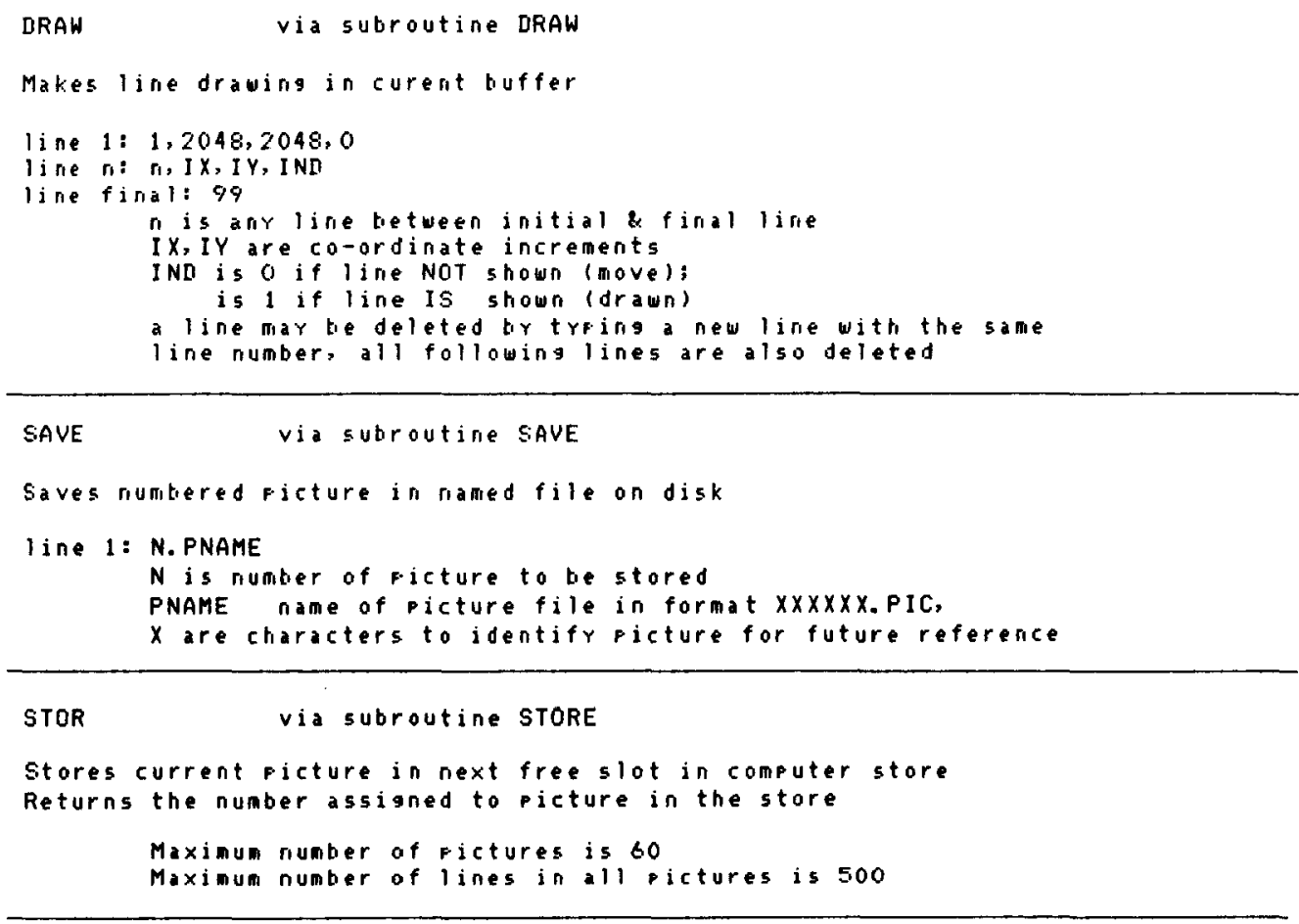

Figure 2. Instructions for commands DRAW, SAVE, STOR. Material that appears on a single index card is delimited by horizontal lines. 


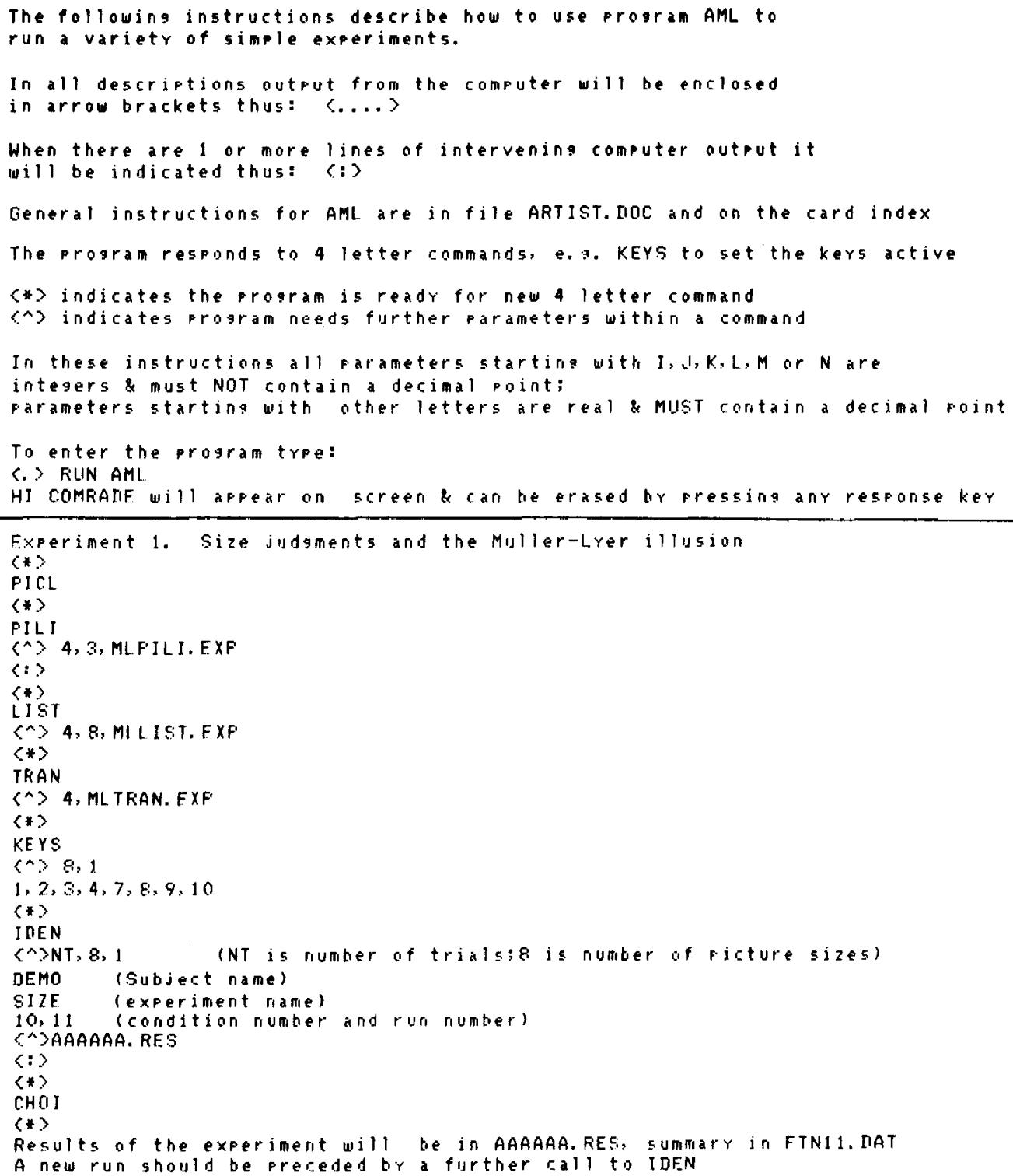

Figure 3. General demonstration instructions and instructions for Mueller-Lyer experiment. Material that appears on a single index card is delimited by horizontal lines.

The second level of user could go on to change the parameters of a demonstration experiment by getting a copy of the specification files used in the demonstration and making similar files with his own pictures, sizes, and so on. The actual specification files for the Mueller-Lyer experiment are shown in Figure 5.

If the required experiment lies outside the demonstration paradigm (or one of the other existing paradigms), then the user will have to do some FORTRAN programming. Users who wish to have access to the full power of the ARTIST system will construct their own experiment running Subroutine CHOICE. They may use a special file, FRAME.FOR, which contains that part of a control subroutine common to all experiments, that is, common blocks for entities used in other parts of the system and initiation of disk files for results and summaries (typically, between 50 and 150 lines of FORTRAN will be needed). Altematively, users may use subroutines of the ARTIST system in programs of their own devising.

\section{IMPLEMENTATION}

The ARTIST package is written in FORTRAN and comprises (1) the main program (194 lines), (2) the general subroutines listed in Table 1 , (3) a number of experimental control subroutines, including the demon- 
stration subroutine in ML.FOR (89 lines) and the effectively empty file FRAME.FOR (28 lines), and (4) a suite of MACRO (machine language) routines,

Table 1

ARTIST Subroutines and Their Functions

\begin{tabular}{llrl}
\hline Subroutine & \multicolumn{1}{c}{ Function } & L & p \\
\hline ASSEMB & $\begin{array}{l}\text { assembles picture with transforma- } \\
\text { tions from computer store to }\end{array}$ & & \\
& $\begin{array}{l}\text { display buffer } \\
\text { flash clears screen }\end{array}$ & 53 & No \\
DLEAR & adds a line to a picture & 41 & No \\
LISTER & accepts text from disk or terminal & 15 & Yes \\
MOMENT & calculates first four cumulants, & & \\
& skew, and curtosis of distribution & & \\
& from sums of powers of distribution & & \\
PILIST & variable & 36 & Yes \\
READ & accepts list of pictures & 21 & Yes \\
SAVE & reads picture file from disk & 16 & No \\
SELECT & saves picture on disk & 19 & No \\
& chooses design indexes according to & & \\
SPECS & preset experimental design & 26 & Yes \\
& accepts list of experimental speci- & & \\
STIM & fications from disk or terminal & 39 & Yes \\
STOR & presents stimulus display & 22 & No \\
TEXT & stores picture in computer & 14 & No \\
\hline
\end{tabular}

Note $-L=$ length $P=$ portable. written by the Computer Centre, to control the periph. erals of our system. FORTRAN was chosen because it is very well supported by the manufacturer (unlike PASCAL at the time) and it is compiled (unlike BASIC), so that we could be completely sure of the timing of all machine language subroutines. These considerations outweighed major disadvantages, namely, that modular construction is very cumbersome since arrays have to be dimensioned in all subroutines and the string handling is ghastly. If we were making the decision today, PASCAL would probably be chosen.

Design decisions were guided by the results of empirical research on the psychology of computer programming and on the using of packages. Recent reviews of such work are given by Cuff (1980), Green (1980), Hammond, Long, Clark, Barnard, and Morton (1980), and Schneiderman (1977). The fundamental decision to make the system interactive and command based was made at the outset. When designing the command structure and details, we paid particular attention to aspects of the system concerned with "defaults" and "coherence," since both these factors have large effects on the usability of any system.

As far as defaults are concerned, every parameter of the system that relates to the generation phase is assigned to a default value on entering the program. Thus,

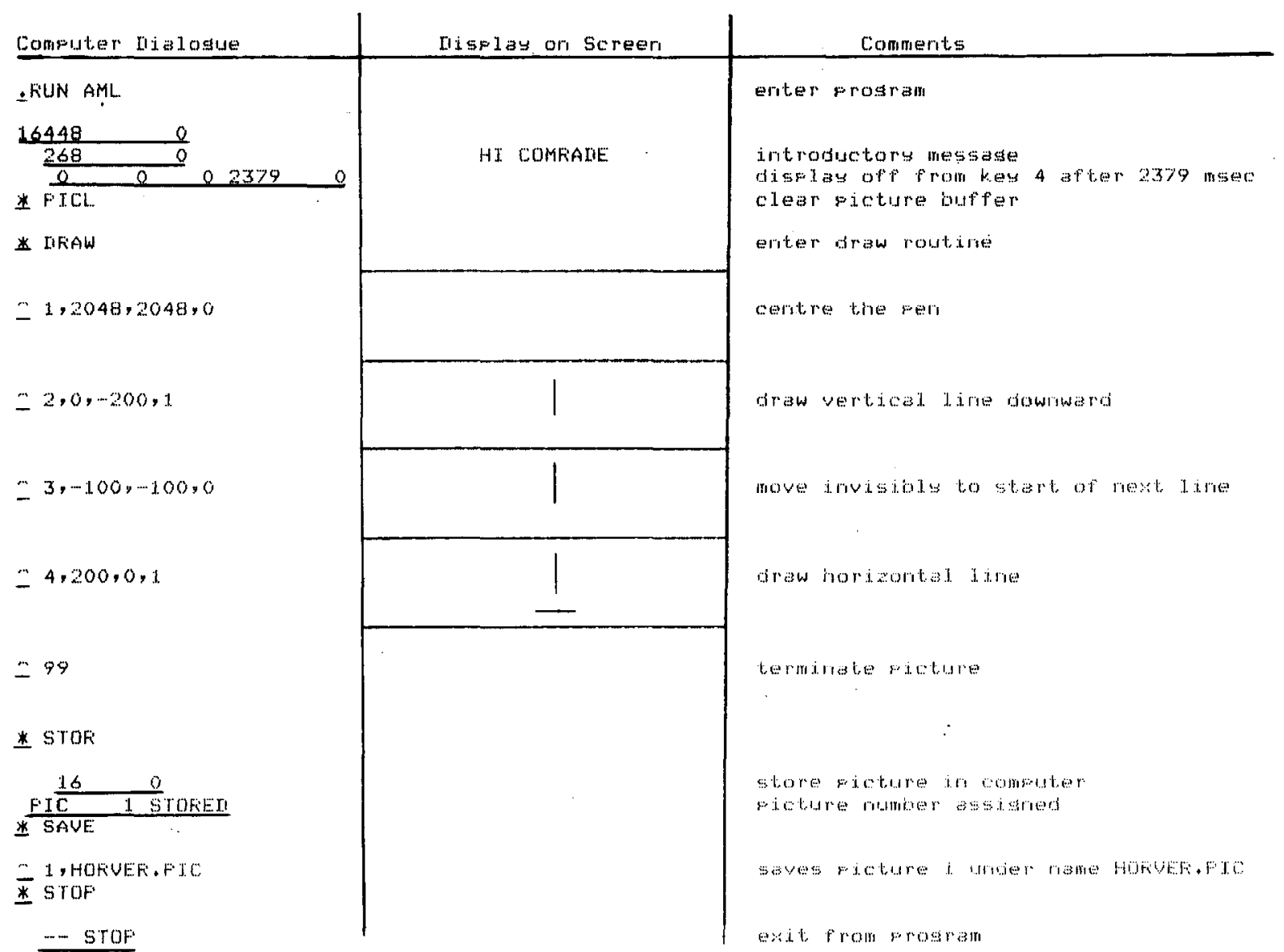

Figure 4. A sample of computer dialogue in the generation phase of ARTIST. Dialogue output by computer is underlined. 


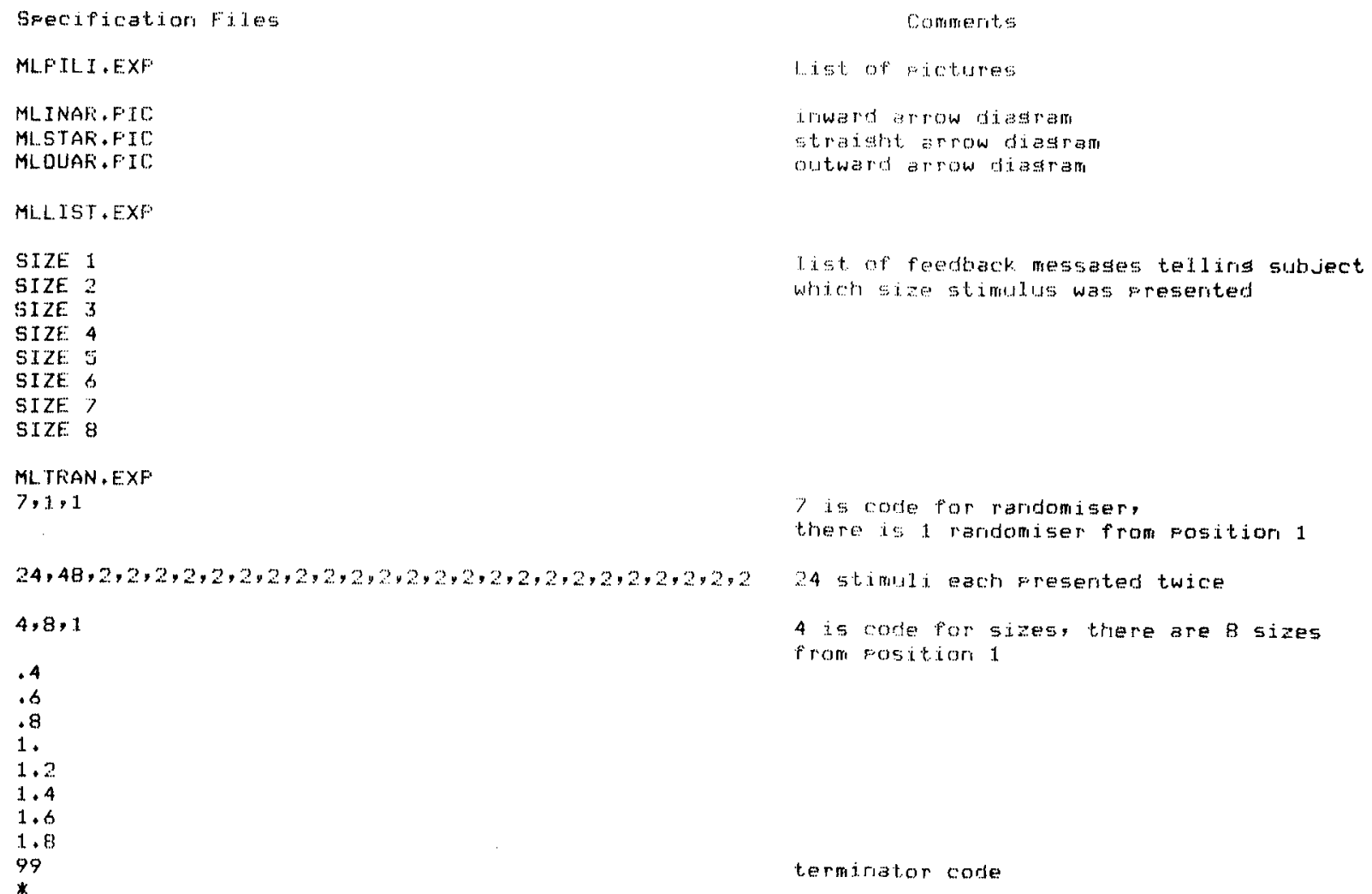

Figure 5. Listings of the specification files for the Mueller-Lyer demonstration experiment.

initially, the current picture buffer contains "HI COMRADE," and all keys are active. The user sees the current buffer displayed and can terminate with any keypress to ensure that the whole system is working at the outset. The second default principle is that every. thing stays as it is until specifically altered. Thus, if TEXT is used to change the size of the letters to double standard size, then letters remain double size until specifically reset to some other size.

"Coherence" must be a somewhat subjective notion. What has been attempted here is to keep parameters that seem to "belong" to the same entity within the same command. For example, TEXT allows the user to enter any character string into the current buffer and to change the size and location of the text, given a continuation code. Clearly, TEXT could have been split into two commands. However, we chose to keep the two parts together for the sake of "coherence." Furthermore, relating back to the default issue, since the characters are changed far more often than are size and location, the same size and location are continued by default.

\section{EVALUATION}

In evaluating the system, we will first look at the implementation phase and then proceed to the use of the package in our department.

\section{Implementation}

The main package was created in several periods of intense activity. The first was in spring, 1979, and that version was used by one graduate student. The next period was in the winter, 1979-1980, resulting in a working system including demonstrations. Effectively, the system has been in use since January 1980 .

The author spent, overall, about 5 months on the project at perhaps $16-20 \mathrm{~h} /$ week. During this time, nearly 1,000 lines of code were written. Several problems were encountered. A major problem was space: With 24K memory, "friendly prompts" had to be kept to a minimum, and most of the instructions and specifications had to be on the card index system rather than immediately available from the computer. A physical problem was the Tektronix oscilloscope, which flickered with large pictures or text.

\section{ARTIST in Use}

Our criteria for evaluation of the system in use were simple: First, does the package do the required task? Second, how much time and effort does the implementation of each new project take?

The demonstration package is evaluated first. It is supposed to acquaint a potential experimenter with the power of the system and also to provide a jumping-off point for new projects. The demonstrations currently 
implemented are the Mueller-Lyer experiment already described, a word classification task, and a replication of an experiment by Sternberg, Monsell, Knoll, and Wright (1978) on the latency and duration of rapid movement sequences. The demonstrations certainly evoked interest and caused some people to use the system; how effective they were as a training tool is yet to be systematically investigated.

At the next level of complexity, the package was used in spring, 1980, for a 2nd-year laboratory exercise, replicating an experiment of Stanovich and Pachella (1977) on the effect of response modality (voice or keypress) and stimulus frequency on choice reaction time in an eight-choice task (identify the numerals 1-8). As far as implementation was concerned, the experiment took only about $1 \mathrm{~h}$ to set up, since it fit the single-interval paradigm of the demonstration subroutine. The experiment was successful with respect to leaming psychology, but it was more problematic with respect to learning the system. Only 1 of these 28 students has so far chosen to use the system for his final-year project. The reasons for this limited success probably have to do with both the fact that students had only about $2 \mathrm{~h}$ on the system (not enough to satisfy the twin goals of doing the experiment and getting well acquainted with the system) and the fact that the documentation was incomplete at that stage.

Two final-year projects have used the system. The first, in $1979 / 1980$, was actually implemented by the author, according to the specification of the student, and involved a word classification task in which some words were laterally inverted (mirror images). The student was able to use the generation phase to decide on the parameters of stimuli, and she also constructed all her own stimulus files using the editor. The system certainly performed the task and took about $4 \mathrm{~h}$ to implement. The second student, who had used the system in the previous year, is investigating visual search time as a function of number of elements and the category of targets and distractors. He successfully wrote his own program, using the ARTIST subroutines SELECT to randomize stimuli and STIM to present them.

The package has also been used by a postgraduate student investigating imagery and memory. This project has not been so successful; the student took an early version of the package and modified it extensively. However, the rather complex line drawings were not really adequate, partly due to the limitations of the display unit.

The author has so far implemented two series of research experiments. The first series comprises arithmetic tasks, in which subjects have to add and compare numbers that include either decimal or fractional parts. The nine numerals in each problem are generated randomly according to rather complicated constraints in a two-factor design. The control subroutine took 10 days to write at a minimum of $10 \mathrm{~h} /$ day. The difficulty occurred because the randomization problem itself was complex and, effectively, the difficulties were being worked out as the program was written. However, once the main control program was written, new conditions could be implemented in about $10 \mathrm{~min}$, using the editor to construct the specification files. The second series of experiments involves signal detectability theory tasks with payoffs for different response-stimulus contingencies and penalties that either are a function of latency or occur whenever the subject exceeds a deadline. The initial experiment took about 1 day to implement, plus a further half-day to implement the deadline conditions. Again, the system was able to perform the task and the time for implementation was small, relative to the time taken to design the experiment.

Projects currently being implemented include an experiment on interresponse times in movement sequences and a general staircase method for thresholds.

All the users described so far had some verbal description and demonstration of the system from the author. The documentation has also been assessed by a single graduate student, who learned the system entirely from the written documentation. Many improvements have been made in the light of his experiences. Nevertheless, he was able to use all the commands for the generation phase and to not only run the demonstrations but also modify them to create new experiments.

\section{DISCUSSION}

The ARTIST system is a computer tool within a single psychology department. However, in the discussion of the system, those aspects that may be of use outside the home department will be emphasized.

At the most concrete level, Table 1 indicates which subroutines are directly transportable. SELECT and MOMENT are the ones likely to be of most use to psychologists. SELECT chooses a random order of stimulus presentation for any factorial design; indeed, it may be used to generate such a random order for an experiment not run on a computer. MOMENT is an analysis subroutine. The fact that it operates on sums of observations $\left(\Sigma x, \Sigma x^{2}\right.$, etc.) means that it is very easy to combine data from different experimental runs.

The entire system should be transportable to any computer with a FORTRAN compiler. Only those routines that control the peripherals would need to be rewritten. ${ }^{1}$

At a more general level, ARTIST may be compared with other packages. Any of the teaching experiments with visual stimuli described by Fischler (1980) and White (1980) could be set up within a half-day session, as could most of the visual research experiments described by Poltrock and Carr (1980) (the ones using rotation and projection of three-dimensional figures would take longer).

There are also several similarities between ARTIST and commercially available packages such as word 
processors. For example, many word processing packages are, like ARTIST, command based. This gives the advantages of an interactive system, namely, that the result of each user action may be seen immediately and the system is modular in the sense that users need only learn about commands relevant to their own needs.

In another sense, ARTIST is very different from commercial packages, most of which are designed to be completely self-contained so that the user interacts only with the package, never with the main computeroperating system behind it. The ARTIST system, on the other hand, makes maximum use of the powerful system facilities embodied in the editor and file manipulation routines. There are two reasons for this design decision. First, no time is wasted duplicating existing facilities that are already tried and tested. Second, more of what the user learns is generally applicable. Students who move to another department or from the laboratorycontrol computer to the main timeshared system will certainly need to know about editors, whereas they are unlikely to encounter another ARTIST.

Considerable attention has been paid not only to the system itself, but also to the supporting documentation. The documentation, like the system, is modular, so that users need look at only one command at a time. The relevant information is also much easier to find from a card system than it would be from a booklet. The documentation is designed so that evaluation and improvement constitute an ongoing process. Users are encouraged to clip any comments or suggestions to the relevant instruction card. Because of the modular construction, the programmer may then modify instructions to just one card and try the new version on another user. If more space were available, these features could be implemented on-line. That is, users would be able to ask for help on any command and also write comments on any command.

The system is designed so that the user can "learn by example." Thus, the immediate availability of specification files for all existing experiments is an essential feature of ARTIST. Again, the value of this approach is a matter for future empirical test.

ARTIST is perhaps unusual in that it deliberately allows for users of different levels of sophistication. Nevertheless, before running any experiment, the user must invest some appreciable time in learning the system. It is hoped that the time is small enough that the exercise is worthwhile even for users who use the system only once.

It is important to identify those features of the package that contribute substantially to a reduction in the labor required to implement an experiment. First, the package almost eliminates the need to write input/ output routines, which can be as much as a third of a program. Similarly, pictures can be created interactively and without programming. The facility of accessing long lists of parameters from disk as well as terminal also saves time, not only when creating experiments but also each time they are used. The presence of a laboratory system like ARTIST permits the buildup of a library, so that most new experiments will conform to an existing subroutine. It should be emphasized that if a library routine exists, an experiment can be set up in half a day. Neither alternative equipment (tachistoscopes?) nor an outside systems analyst's help could improve on this time. It is quite impossible to write a package that will cover all possible future experiments, a fact that must be appreciated by psychologists and the computer experts they commission. Hence, the facility to write user programs in a widely available and well supported language that can easily be linked to the package is essential.

Finally, it must be emphasized that techniques for evaluating complex systems or packages are in their infancy. Consequently, the above discussion is based, at best, on empirical field observation, but, more frequently, on the intuition of the author or the users.

\section{REFERENCES}

CufF, R. N. On casual users. International Journal of ManMachine Studies, 1980, 12, 163-187.

Fischler, I. An on-line laboratory in cognition and perception. Behavior Research Methods \& Instrumentation, 1980, 12. 116-119.

GreEN, T. R. G. Programming as a cognitive activity. In H. T. Smith \& T. R. G. Green (Eds.), Human Interaction with computers. London: Academic Press, 1980.

Hammond, N., Long, J., Clark, I., Barnard, P., \& Morton, J. Documenting human-computer mismatch. Proceedings of the Ninth International Symposium on Human Factors in Telecommunication, Red Bank, New Jersey, 1980, 9, 17-24.

Poltrock, S. E., \& CARR, W. G. A flexible computer-controlled perception laboratory. Behavior Research Methods \& Instrumentation, 1980, 12, 132-136.

SChNEIDERMAN, B. Measuring computer program quality and comprehension. International Journal of Man-Machine Studies, $1977,9,465-478$.

Stanovich, K. E., \& Pachella, R. G. Encoding, stimulusresponse compatibility and stages of processing. Journal of Experimental Psychology: Human Perception and Performance, $1977,3,411-421$

Sternberg, S., Monseld, S., Knoll, R. L., \& Wright, C. E. The latency and duration of rapid movement sequences. In G. E. Stelmach (Ed.), Information processing in motor control and learning. New York: Academic, 1978.

White, $K$. D. An on-line laboratory in sensation and perception. Behavior Research Methods \& Instrumentation, 1980, 12, 114-115.

\section{NOTE}

1. The following material is available from the author on any computer medium: (a) any subroutine, (b) the entire ARTIST system, and (c) ARTIST documentation. All subroutines marked portable in Table 1 will run on any FORTRAN machine and are fully supported; the rest of the system is guaranteed to work only on systems with hardware configuration identical to ours. 